will be asked, How are these observations made, and how $\mathrm{i}$ : it known when the star is in the same position when the second observation is made?

For this purpose a transit instrument is used (see Fig. 30). This differs from an ordinary telescope, being so mounted as to move only up and down, and is armed not with simple cross wires, but with an odd number of parallel and equidistant vertical wires crosced by a single horizontal wire. It is also usually proi:ded with a circle to give declination. If from any part of the earth an observation be made on any particular star on one day, and then another observation made on the same star when it is in the same position the next day, as has been said, the interval between the two observations must be the time taken by the earth to move round once.

By having such an arrangement as exists in the transit instru-

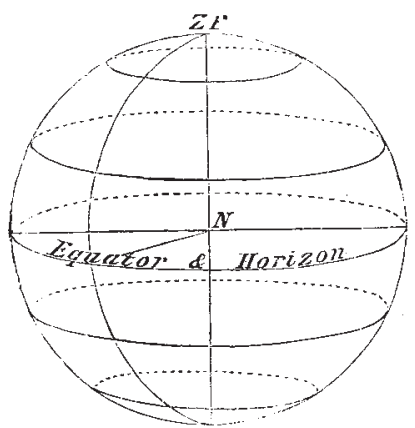

FIG. 32.-Showing that the true horizon of a pole is the equator.

ment, by which it can swing in the plane which coincides with the axis on which the earth turns, any star may be chosen for the observation. Suppose, for instance, the instrument be pointed to the north pole star, then, in cqnsequence of the tremendous distance of the stars, the axis of the telescope is practically coincident with the axis of the earth. But suppose another star to be observed, it will be quite clear that we may make the observation on it, or any other star we cboose. When the instrument is upright it points to the zenith. A star in the zenith may therefore be selected for the observation.

It is observed when crossing the central wire of the instrument one day, and noted again when it crosses that wire on the succeeding day. But the observer does not limit his observation to the one central wire, in order to ascertain when the star is in the centre of the field. If he did so, he might miss his observa-

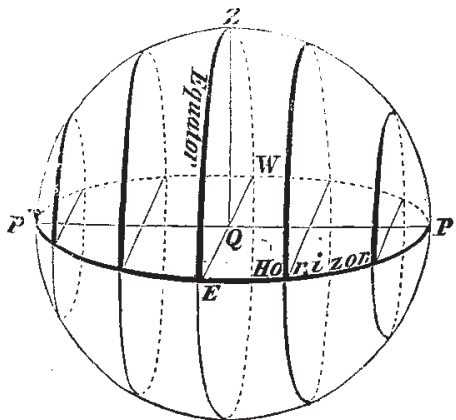

Fig. 33,-Showing that the poles lie in the horizon at the equator.

tion. That is why the simple cross wires have been replaced by a system of wires (see Fig. 3I). As the star crosses the field of view, the observer, listening to the beats of the clock alongside, notes the time when it crosses each of the wires, and takes the mean of these observations, thus attaining to a much greater accuracy than if he had merely observed the transit over the central wire. With an ordinary clock it is found that a perio 1 , less by a few moments than twenty-four hours, elapses between two successive transits.

In order to get an absolutely perfect measure of time, the clock may be so rated that it should not be any indeterminate number of hours, minutes, and seconds, but twenty-four hours exactly between the two transits of that star. With a clock thus arranged, the time at which a star crossed the central wire of the transit instrument would really give a most perfect method of determining that star's place in the heavens, because, if the earth's rotation is an equable one and takes place in a period which we choose to call twenty-four hours, then two stars $180^{\circ}$ apart will be observed twelve hours after one another, four stars $90^{\circ}$ apart will be observed six hours apart, and so on; and clocks like this, regulated to this star time, exist in our observatories, being called sidereal clocks, because the time they give, which is not quite familiar to everybody, is called sidereal time.

Now let us consider our position on the earth with regard to the stars. This is a very interesting part of our subject, not only in its scientific aspect, but from the point of view of its usefulness, whether we wish to study the stars or define places on the earth's surface, the latter matter, however, being so intimately connected with astronomy proper that it is impossible to talk about the one without talking about the other.

Since we divide all circles into $360^{\circ}$, the circumference of the earth may be so divided, and the method in use of defining positions on the earth is to say of a place that its latitude is so much and its longitude is so much. Latitude begins at the equator with $0^{\circ}$, and terminates at the poles with $90^{\circ}$, being north latitude in the one case, and south latitude in the other. In the case of longitude, there is no such simple starting point, for whilst latitude is counted from the equator by everybody all over the world, longitude may commence at any point. In England we count longitude from the meridian of Greenwich. When the transit instrument at Greenwich is swept from the north point through the zenith to the south point it describes a half circle, which is called the meridian of Greenwich.

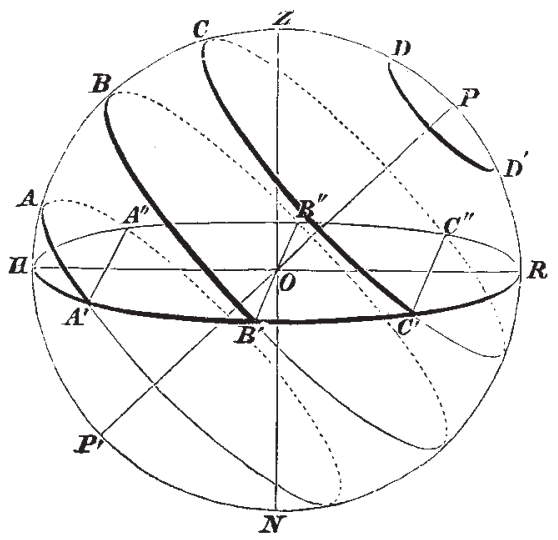

FIG- 34.- Horizon of a place in mid-latitude,

That is one point. Another point is this. Suppose the instrument to be set up not at Greenwich but at the north pole. Then the true horizon of the observer will be along the equator. Remove the instrument to the equator, and the true horizon will cut the poles. At a place in mid-latitude the true horizon would cut neither the pole nor the equator, but would be inclined to both (see Figs. 32, 33, and 34).

Then comes the important relationship between the latitude of the place and the altitude of the pole star above its borizon; that the number of degrees this star-be it north or south-is above the horizon of the observer will be the number of degrees of north or south latitude of the place where the observation is made. A place therefore in $10^{\circ} \mathrm{N}$. lat. will (ronghly) have the north pole star at a height of $10^{\circ}$ above its horizon.

So much for this part of our subject. Let us now leave it, because, interesting as it is, it refers to a branch of astron my with which at present we have less to do than with the $m$ re physical one; but it was well that we should pause for a few moments to note the tremendous importance to mankind of that particular movement of the earth which we have been considering

(To be continued.)

Norman Lockyer

\section{PROBABLE NATURE OF THE INTERNAL} SYMMETRY OF CRYSTALS

THE theory of the modification of crystal angles, just offered in dealing with quart $z$, is manifestly applicable to all crystals not of the cubic system, and it is submitted that for every suck ${ }^{x}$ Continued from p. $x 88$. 
crystal there is an ideal or root form proper to one or other of the five kinds of internal symmetry which have been presented, from which root form the actual form can be derived by a proper proportionate increase of dimension in one or more directions.

It is evident that, while our rath must become more and more intricate as we endeavour to establish in the cases of more complex compounds relations similar to those above traced, the reference of whole classes of analogous forms, differing only in their angles, to one root form, removes a very important difficalty, and the wide applicability which it confers on the five kinds of internal symmetry with which we started appears in the fact that there is no crystal form which cannot be thus referred to an appropriate root form in harmony with one or other of these five kinds of internal symmetry. ${ }^{1}$

One more case may be mentioned in which a probable internal symmetry can be assigned to a compound in harmony with its actual crystal form; it is a more difficult one.

The molecule of Iceland or calc-spar is usually believed to consist of one atoin of calcium, one atom of carbon, and three of oxygen. We shall, however, take a liberty, and suppose that the atoms of calcium or the atoms of carbon have but half the mass attributed to them; that in the formula of this compound we should write either two atoms of calcium or two atoms of carbon in place of one. ${ }^{2}$

Making this supposition, we observe that if the calcium and carbon atoms were alike we should have six atoms, three of one kind, three of another; in other words, we should have equal proportions of two linds of atoms, from which, since the form of Iceland spar is but little removed from a cube, we naturally argue that just before crystallisation its atoms were arranged according to the fir-t or second kind of internal symmetry ; these two kinds being, it: will be remembered, those in harmony with the cubic form which admit of very symmetrical arrangement of particles of two kinds present in equal numbers.

Since-Iceland spar is a uniaxal crystal, the arrangement of the three kinds of atoms, whatever it is, must be symmetrical about one axis only; and we shall now endeavour to show that the atoms can be thus arranged in either the farst or second kind of symmetry.

We will show first that they can be hus arranged in the second kind.

Where there a:e but two kinds of particles present in equal numbers, symmetry requires that the alternate layers of this kind of symmetry (see Fig. 3) shall consist entirely of similar kinds, and therefore in the case before us, one set of alternate layers will represent oxygen atoms ; the other, atoms of calcium and carbon. Now particles present, as we suppose the calcium and carbon atoms to be, in the proportion $I: 2$ can be quite symmetrically arranged in these layers (plan $f$ ), as the sphere centres were in the layers depicting the fourth kind of symmetry (plan e), and therefore the only question remaining is the relative disposition of the layers of calcium and carbon atoms with respect to one another.

Now the spheres in alternate layers of the second kind of symmetry considered alone have the relative arrangement of the third kind of symmetry (Fig. 4), and in determining the relative disposition of the calcium and carbon atoms, we may therefore neglect the oxygen atoms, and treat the case as belong. ing to the third kind of symmetry. The two spiral arrangements in this kind of symmetry, in which the less numerous spheres in the fourth layer are vertically over those in the first (see ante), have the necessary symmetry about a single axis, and if the calcium and carbon atoms have one of these arrangement;, the requirements of the case are entirely met.

We will now show that the three kinds of atoms can also be arranged symmetrically about a single axis in the first kind of symmetry.

One hali the spheres depicting this kind of symmetry will in this case represent the oxygen atoms, and the remaining half the atoms of calcium and carbon (see Fig. 2), and, as previously noticed, the arrangement of either half will be that of the second kind of symmetry. It follows that the question of the relative disposition of the atoms of calcium and carbon is simply the question of the symmetrical arrangement about a single axis of atoms of two kinds present in the proportion $2: I$ in the second

I The very symmetrical form the pentagonal dodecahedron is not in harmony with either of the five kinds of symmetry, nor is it found in crystals.

It has already heen remarked that the crystal form of fluor-spar favour the supposition that calcium has half the atomic weight usually attributed to it. kind of symmetry (Fig. 3). And since the layers of spheres depicting this kind of symmetry have a triangular arrangement (plan b), it is evident that this can be accomplished here juit as in the former case.

In tither of the two arrangements just described we have only to suppose that when the symsaetrically placed atoms change volume at the time of crystallisation the dimensions transversely to the axis of symmetry are increased relatively to those in the direction of this axis, and we have an obtuse rhombohedron where formerly we had a cube. And the significant fact that the angle of a rhombohedron of calc-spar diminishes when the crystal is heated supports this theory of its production. Perhaps the arrangement of the atoms according to the first kind of internal symmetry is the more probable of the two, as this would give the cleavage directions coincident with the directions of layers of similar atoms (oxygen).

An important fact supporting our conclusions is that certain definite relations as to their proportions which are found subsisting between the allied forms taken by crystals of the same substance are found inherent in one or other of the five kinds of internal symmetry.

Thus it is well known that if a particular substance is found crystallised in hexagonal pyramids of various linds-that is, whose sides have various different degrees of inclination to the base-the number of kinds is strictly limited, and they are strictly related to each other. If $x$ be the side of the hexagonal base of the pyramid and $y$ the height for the same substance, while $x$ remains constant, $y$ has not more than fourteen different values, seven related thus: $c, \frac{3}{4} c, \frac{1}{2} c, \frac{1}{4} c, \frac{1}{8} c, \frac{1}{12} c, \frac{1}{16} c ;$ and the other seven sinilarly related thus : $d, \frac{3}{4} d, \frac{1}{2} d, \frac{1}{4} d, \frac{1}{8} d, \frac{1}{12} d, \frac{1}{16} d$; and $c$ bearing to $d$ the ratio $2: \sqrt{ } / 3$.

Now, if we turn to the fourth lind of internal symmetry (Fig. 5) to ascertain the possible varieties of inclination of the sides of hexagonal pyramids which can be depicted, we find that the greatest possible height to which we can build a hexagonal pyramid of equal spheres is exactly double the height of a tetra. hedron with the same side as the hexagonal base of the pyramid. Thus, if twenty-five spheres form each side of the hexagonal base, giving twenty-four equal distances between the sphere centres in any one side, we find that the highest possible pyramid has forty-nine layers of spheres giving forty-eight equal spaces between concecutive layers.

If we call this height $c$, it is evident that pyramids corresponding with the first of the above series of actually observed forms wil have respectively-

49 layers of balls, giving 48 spaces between consecutive layers. 37 25

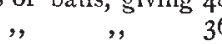

, $\quad$," 40,36,

" $24 \quad$ " 12 ,

,

" " 4

We find, moreover, that such a series can be readily depicted, and that, upon examination, no additional terms appear admissible.

Again, a further inspection of the stack of spheres shows us that with the same heights-that is, with the respective numbers of layers just enumerated-we may, in place of the base layer which forms a hexagon whose sides have twenty-five spheres each, have a base derived from this in which each of the six spheres at the angles becomes the centre of a side, the outline of the base layer being now a larger hexagon described about the hexagon which bounded the former base layer. The sides of this new base thus bear to the sides of the old the ratio subsisting between the side and the perpendicular of an equilateral triangle, i.e. the ratio $2: \sqrt{ } 3$. And finally, since the distance between the planes containing the centres in suceessive layers bears to the distance between centres in the same layer the same ratio which the perpendicular from the angle of a tetrahedron upon its opposite face bears to its edge, that is the ratio $\sqrt{2}: \sqrt{3}$, it follows-

That the two allied series of possible altitudes of hexagonal pyramids thus formed, if we take the same length of sicie $a$ for both, will be--

$$
\begin{aligned}
& \frac{2 \sqrt{2}}{\sqrt{3}} a ; \frac{3 \sqrt{ } 2}{2 \sqrt{ } 3} a ; \frac{\sqrt{2}}{\sqrt{ } 3} a ; \frac{\sqrt{2}}{2 \sqrt{ } 3} a ; \frac{\sqrt{2}}{4 \sqrt{ } 3} a ; \frac{2 \sqrt{ }}{6 \sqrt{3}} a ; \frac{\sqrt{2}}{8 \sqrt{ } 3} a . \\
& \text { Second Series }
\end{aligned}
$$


Surely the fact thus established, that each term of a series of relative altitudes of the hexagonal pyramids in which a particular substance crystallises always has to some term of the series thu: theoretically derived a particular ratio peculiar to the substance, constrains us to conclude that the above fourteen "root" forms are those to which all crystal forms involving regular six-sided pyramids are referable, and that the actual forms are produced from the "root" forms by difference in the degree of expansion in the direction of the axis of the crystal as compared with other directions at the time of crystallisation.

Other allied forms, as allied octahedra or rhombohedra, can be in the same way connected with some one of the five kinds of internal symmetry.

The peculiarities of crystal-grouping di iplayed in twin crystal can be shown to favour the supposition that we have in crystals symmetrical arrangement rather than symmetrical shape of atoms or small particle.. Thu; if an octahedron be cut in half by a plane parallel to two opposite faces, and the hexagonal faces of separation, while kept in contact and their centres coincilent, are turned one upon the other through $60^{\circ}$, we know that we get a familiar example of a form found in some twin crystals. And a stack can be made of layers of spheres placed triangularly in contact to depict this form as readily as to depic a regular octahedron, the only modification necessary being for the layers above the centre layer to be placed as though turned bodily through $60^{\circ}$ from the position necessary to depict an octahedron (compare Figs. 7 and 8 ). The modification, as we see,

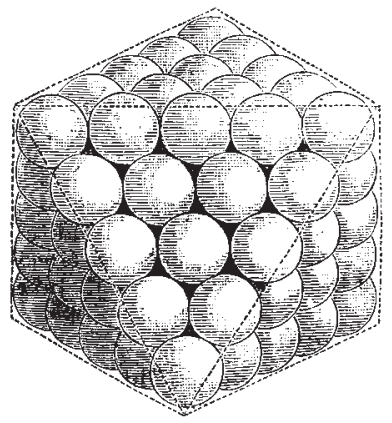

FIG. 7.

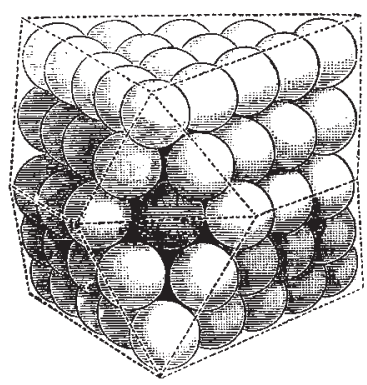

FIG. 8. involves no departure from the condition that each particle is equidistant from the tzelve nearest paricles.

Before closing, a few words may be said on the bearing of the conclusions of this paper on isomorphism and dimorphism.

First, as to isomorphism.

The conclusion that there are but five kinds of internal symmetry possible, three of which indicate a cubic form, evidently accords with the fact that not only the simplest combinationsthose in which two kinds of atoms are present in equal proportions-but also many very complicated compounds crystallise in cubes.

Out of the regular system we generally find that for the angles of crystals of different compounds to be the same there must be some resemblance in their atom-composition, and the explanation suggested is that the atoms which are common to two isomorphous compounds, $e . g$. the carbon and oxygen atoms in calcspar and spathic iron ore, have similar situations in the two different crystals, and that the change of bulk which occurs when crystallisation takes place is due to a change in these atoms only, the atoms not found in both remaining passive.

There are, however, some cases which do not at first seem to be met by this view-cases in which the atom composition of isomorphous compounds has only a very partial similarity. Ammonia compounds may be specially mentioned. Thus, ammonic sulphate, $\left(\mathrm{NH}_{3}\right)_{2} \mathrm{H}_{2} \mathrm{SO}_{4}$, is isomorphous with potassic sulphate $\mathrm{K}_{2} \mathrm{SO}_{4}$.

The following suggestion would seem to enable us to suppose that in this, as in other cases of isomorphism, the phenomenon is referable to the passivity of some of the atoms in the change of bulk which accompanies crystallisation, Let us write ammonic sulphate thus $\left(\mathrm{NH}_{3}\right)_{2} \mathrm{H}_{2} \mathrm{SO}_{4}$, and let us suppose that the symmetrical arrangement is such that the groups, $\left(\mathrm{NH}_{3}\right)_{2}$ just occupy places which might, without altering the symmetry, be filled by additional groups $\mathrm{H}_{2} \mathrm{SO}_{4}$; that, in other words, the relative position of the groups $\mathrm{H}_{2} \mathrm{SO}_{4}$ which are present in the symmetrical arran rement is precisely the sume as it would be if the entire mass consisted of these group; instead of consisting partly of $\mathrm{NH}_{3}$ groups. If now, in aldition to supposing that in both compounds the aclive atoms in the process of crystallisation are the sulphur and oxygen atoms, and these only, we sup. pose that the expansion of sone of the atoms of the active kind checks the expansion of others; that only a certain proportion of these atoms expands, we perceive that we may have both the same amount and lind of atom expansion in the two cases, and, as the natural result, isomorphism.

Next, as to dimorphism.

It is evident that a very small change is requisite to convert one kind of internal symmetry into another. Thus we have already had occasion to notice that the only difference in depicting the third and fourth kinds of symmetry is that for the former the centres of the spheres in the first and fourth layers, those in the second and fifth, and so on, range vertically, while for the latter the centres in the first and third, in the second and fourth, and so on, range in this way.

In the case of a dimorphic compound consisting of two kinds of atoms in the proportion of $2: \mathrm{I}$, e.s. water, $\mathrm{H}_{2} \mathrm{O}$, we have only to suppose therefore that the same layers of atoms which under one set of conditions produce hexagonal prisms, are by some alteration in conditions arranged in the slightly different way necessary to produce rhombohedral forms. Other cases of dimorphism are probably to be accounted for much in the same way.

Thus the following interpretation of the fact that calcic carbonate, which we have seen crystallises in obtuse rhombohedra as calc-spar, sometimes crystallises in six-sided trimetric prisms as aragonite may be offered.

We have already endeavoured to show that the first or second lind of internal symmetry is that proper to calc-spar. We will now endeavour to show that the fifth kind of internal symmetry (Fig. 6) is proper to aragonite.

Alternate layers of spheres (plan $b$ ) will represent the oxygen atoms, and the other alteriate layers the calcium and carbon atoms; the central layers of the triplets above alluded to, viz. the second, the fourth, the sixth, \&c., being the oxygen layers ; the calcium and carbon atoms in the ramaining layers will be symmetrically arranged (plan $f$ ). From the fact of the crystals being trimetric, the layers containing the last-named atoms, whicb, considered apart from the oxygen layers, are in the fourth kind of symmetry, probably have the arrangement above described, in which the less numerous spheres form zigzags, the stack in this case having a different symmetry about three axes at risht angles to each other (Fig. 6).

The fact that the dimorphic varieties of the same substance have different densities is in harmony with the supposition that different sets of the atoms are concerned in the different cases; that the active atoms which produce one form are not those, or those only, which produce the other.

It is not always necessary to refer two incompatible crystal forms of the same substance to two different kinds of internal symmetry: for example, from the third kind of internal symmetry we can produce square-based octahedra, and we can also produce right-rhombic prisms, and in accord with this we have the well-known fact that right-rhombic prisms of sulphate of nickel, $\mathrm{N}_{2} \mathrm{SO}_{4} \mathrm{H}_{2} \mathrm{O}$, when exposed to sunlight are molecularly transformed, and, though they neither liquefy nor lose their form, when they are broken are found to be made up of square-based octahedra several lines in length.

WILLIAM BARLOW

\section{UNIVERSITY AND EDUCATIONAL INTELLIGENCE}

CAMBridge. - The following awards (among others) have been made at St. John's College on the results of the examination for candidates who have not yet commenced residence :-

For Mathematics: H. F. Baker (Perse Grammar School, Cambridge), Foundation Scholarship, raised for two years to 75l. a year; A. W. Flux (Portsmouth Grammar School), Minor Scholarship of 75 . a year; P. T. Fagan (Highwood School, Weston), Exhibition of 50 . a year; H. R. Norris (University College School), Exhibition of $30 l$. a year.

For Natural Science : G. S. Turpin (Nottingham High School and Owens College, Manchester), Foundation Scholarship raised for two years to $75 \%$. a year ; P. Lake (Newcastle College of Science), Minor Scholarship of $75 l$. a year; W. Harris (Bradford Grammar School), Exhibition of 50 l. a year; W. M. Mee 